La Revue

des Droits

de l'Homme

\section{La Revue des droits de l'homme}

Revue du Centre de recherches et d'études sur les droits fondamentaux

Actualités Droits-Libertés | 2020

\title{
Des mesures de sûreté et des mots
}

À propos de la décision $n^{\circ} 2020-805$ DC du 7 août 2020, Loi instaurant des mesures de sûreté à l'encontre des auteurs d'infractions terroristes à l'issue de leur peine

\section{Patricia Rrapi}

\section{(2) OpenEdition}

\section{Journals}

Electronic version

URL: http://journals.openedition.org/revdh/10472

DOI: $10.4000 /$ revdh. 10472

ISSN: 2264-119X

Publisher

Centre de recherches et d'études sur les droits fondamentaux

Electronic reference

Patricia Rrapi, « Des mesures de sûreté et des mots », La Revue des droits de l'homme [Online],

Actualités Droits-Libertés, Online since 08 October 2020, connection on 06 November 2020. URL http://journals.openedition.org/revdh/10472 ; DOI : https://doi.org/10.4000/revdh.10472

This text was automatically generated on 6 November 2020 .

Tous droits réservés 


\section{Des mesures de sûreté et des mots}

À propos de la décision n²020-805 DC du 7 août 2020, Loi instaurant des mesures de sûreté à l'encontre des auteurs d'infractions terroristes à l'issue de leur peine

\section{Patricia Rrapi}

\section{I/- Bref rappel}

1 Dans la décision $\mathrm{n}^{\circ}$ 2017-750 DC du 23 mars 2017, Loi relative au devoir de vigilance des sociétés mères et des entreprises donneuses d'ordre ${ }^{1}$, le Conseil constitutionnel censurait l'amende prononcée à l'égard des entreprises en cas d'absence de plan de vigilance destiné à identifier les risques et à prévenir les atteintes graves envers les droits humains et les libertés fondamentales, la santé et la sécurité des personnes ainsi que l'environnement :

2 "Compte tenu de la généralité des termes qu'il a employés, du caractère large et indéterminé de la mention des «droits humains » et des « libertés fondamentales » et du périmètre des sociétés, entreprises et activités entrant dans le champ du plan de vigilance qu'il instituait, le législateur ne pouvait, sans méconnaître les exigences découlant de l'article 8 de la Déclaration de 1789 et en dépit de l'objectif d'intérêt général poursuivi par la loi déférée, retenir que peut être soumise au paiement d'une amende d'un montant pouvant atteindre dix millions d'euros la société qui aurait commis un manquement défini en des termes aussi insuffisamment clairs et précis. »

La facilité avec laquelle le juge des droits et libertés qualifie la référence aux «droits humains » et aux «libertés fondamentales » de «large et indéterminée » est moins surprenante que l'énergie avec laquelle le Conseil constitutionnel applique le principe de clarté de la loi. En effet, la censure, fondée sur la violation de l'article 8 de la Déclaration de 1789, suppose au préalable la qualification de l'amende "civile » en " sanction ayant le caractère d'une punition ». C'est parce que l'amende civile est dotée d'une coloration pénale que le principe de clarté de la loi pénale, de même que les autres principes constitutionnels liés à la loi pénale, s'applique dans toute sa rigueur. Le contrôle approfondi, exigeant et protecteur du Conseil constitutionnel est la conséquence de l'élargissement du champ d'application des principes constitutionnels 
de la loi pénale en dehors du droit pénal. Même si, en l'espèce, cet approfondissement du contrôle bénéficie à la protection pénale de multinationales qui n'auraient pas respecté les droits et libertés des femmes et des hommes mobilisés à produire des biens et services pour leur compte, il est, a priori, difficile de ne pas assimiler cette jurisprudence à une meilleure protection des droits et libertés. Cette lecture correspond, en tout cas, au discours qui enveloppe constamment l'élargissement du champ d'application des principes de la loi pénale.

En ce sens, la décision du 23 mars 2017 n'est nullement un cas isolé. Si, compte tenu du contexte social actuel, le Conseil constitutionnel s'est parfois attelé à résister à sa propre jurisprudence ${ }^{2}$, il n'en demeure pas moins généreux. Toute une série de mesures économiques, sociales et fiscales ont été, depuis les années 1980, requalifiées en "sanctions ayant le caractère d'une punition »: la sanction des manquements aux obligations de protection des investisseurs ou au bon fonctionnement du marché3, la sanction des employeurs pour défaut d'accord collectif à l'emploi des salariés âgés ${ }^{4}$, l'amende pour défaut de déclaration de comptes bancaires ouverts, utilisés ou clos à l'étranger ${ }^{5}$, la majoration de la redevance d'occupation du domaine public fluvial pour stationnement sans autorisation, ${ }^{6}$ etc. La requalification pénale desdites sanctions intervient sans tenir compte de la nature juridictionnelle de l'autorité qui les prononce: l'Autorité des marchés financiers ${ }^{7}$, l'Autorité de contrôle des nuisances aéroportuaires $^{8}$, le Conseil de la concurrence ${ }^{9}$, le Conseil supérieur de l'audiovisuel ${ }^{10}$, etc.

\section{II/- Bref étonnement}

Dans la décision du 7 août 2020, les députés et sénateurs auteurs de la saisine ont alors pu légitimement penser que, si un certain nombre de sanctions prononcées par différentes autorités étaient qualifiées de "sanctions ayant le caractère d'une punition ", les mesures de sûreté contestées pourraient l'être également et se verraient appliquer les principes constitutionnels de la loi pénale.

6 En faisant abstraction du caractère particulièrement limitatif de liberté des mesures contestées, la réponse du Conseil constitutionnel, dans la décision du 7 août 2020, comparée à son élan jurisprudentiel en faveur de l'élargissement du champ d'application des principes constitutionnels de la loi pénale, est déconcertante :

7 "Si la mesure prévue à l'article 706-25-15 du code de procédure pénale est prononcée en considération d'une condamnation pénale et succède à l'accomplissement de la peine, elle n'est pas décidée lors de la condamnation par la juridiction de jugement mais à l'expiration de la peine, par la juridiction régionale de la rétention de sûreté. Elle repose non sur la culpabilité de la personne condamnée, mais sur sa particulière dangerosité appréciée par la juridiction régionale à la date de sa décision. Elle a pour but d'empêcher et de prévenir la récidive. Ainsi, cette mesure n'est ni une peine ni une sanction ayant le caractère d'une punition. »

Ce raisonnement résiste à toute logique. Premièrement, un des principaux arguments en faveur de l'élargissement du champ d'application des principes constitutionnels de la loi pénale, est l'absence de caractère déterminant du critère juridictionnel. Le Conseil constitutionnel, lorsqu'il s'agit d'élargir le champ d'application des principes de la loi pénale, rappelle constamment que ces principes s'appliquent «non seulement aux peines prononcées par les juridictions répressives mais aussi à toute sanction ayant le caractère d'une punition ». Dans la décision du 7 août 2020, il mobilise l'argument à 
l'envers. Il décide que la mesure de sûreté contestée ne peut être qualifiée de «peine pénale " précisément parce qu'elle est prononcée non pas par le juge pénal mais par la juridiction régionale de la rétention de sûreté. Le Conseil constitutionnel réserve toute explication sur l'éventuelle distinction qu'il pourrait établir entre le Conseil de la concurrence, par exemple, et la juridiction régionale de sûreté. La seule différence que nous apercevons est fortement inquiétante. Alors que le Conseil de la concurrence et les autres juridictions ou administrations susceptibles de prendre des « sanctions ayant le caractère d'une punition" ne peuvent prononcer, selon les décisions du Conseil constitutionnel, des mesures privatives de liberté, la juridiction régionale de sûreté le peut. Par suite, cela signifie que les principes constitutionnels de la loi pénale peuvent s'appliquer aux mesures non privatives de liberté, à coloration pénale, mais qu'ils peuvent ne pas s'appliquer aux mesures privatives de liberté. Mieux encore, c'est parce que le Conseil constitutionnel refuse de leur appliquer les principes constitutionnels de la loi pénale qu'elles cessent même d'être des mesures privatives de liberté.

9 Ainsi, et deuxièmement, si la finalité de la mesure - prévenir la dangerosité - semble l'emporter sur sa gravité et ses conséquences sur la liberté - la privation -, cela n'empêche aucunement le Conseil constitutionnel de mobiliser, de manière particulièrement spectaculaire, comme fondement constitutionnel de la liberté personnelle, l'article 9 de la Déclaration de 1789 :

10 "Toutefois, bien que dépourvue de caractère punitif, elle doit respecter le principe, résultant des articles 2, 4 et 9 de la Déclaration de 1789, selon lequel la liberté personnelle ne saurait être entravée par une rigueur qui ne soit nécessaire. » (cons. 10)

11 Cet article, selon le Conseil constitutionnel lui-même, pose les principes constitutionnels de la loi pénale et fait, au surplus, expressément référence à la «culpabilité ». Si la mobilisation de l'article 9 de la Déclaration de 1789 peut alors paraître contradictoire, c'est sans doute parce que le Conseil constitutionnel ne peut pas (plus) dire ce qu'il pense.

\section{III/- Zoom sur les mots}

12 Ce 7 août 2020 , le Conseil constitutionnel avait, malgré tout, décidé de censurer les nouvelles mesures de sûreté. Il faut admettre cependant que la tâche était difficile. Non pas parce que la mesure contestée eût été particulièrement protectrice de la liberté. Plutôt parce que le Conseil constitutionnel ayant validé, par le passé, des mesures préventives et limitatives de liberté, notamment les surveillances administratives auxquelles les mesures de sûreté contestées ressemblaient étrangement - ne s'était pas réservé beaucoup d'espace pour introduire des censures.

Premièrement, dans le cadre de la lutte contre l'immigration illégale et, par la suite, dans celui de la lutte contre le terrorisme ${ }^{11}$, le Conseil constitutionnel a validé des sanctions administratives privatives de liberté. Autant par pudeur que par souci d'en transférer la compétence au juge administratif, il a qualifié ces mesures de «mesures restrictives de liberté » et identifié leur finalité comme relevant de la prévention. Du point de vue de la rhétorique des droits et libertés, cette mutation s'est accompagnée par la substitution de la protection des articles 2 et 4 de la Déclaration de 1789 « liberté personnelle» - à la protection accordée par l'article 66 de la Constitution «liberté individuelle»-, article qui aurait conduit, sans ce subterfuge, à la censure pure et simple de telles mesures. En effet, si l'article 66 de la Constitution supporte les 
mesures privatives de liberté, il exclut totalement la compétence des autorités autres que le juge pénal. Ainsi, au moment même où, au nom de la protection des droits et libertés, le Conseil constitutionnel élargissait le champ d'application des principes constitutionnels de la loi pénale, il autorisait l'infiltration du modèle de police administrative dans ce qui aurait dû, en principe, relever précisément du droit pénal. L'argument de la finalité de la mesure, sur les ruines de celui de la gravité de celle-ci, lui a ainsi permis de fabriquer au niveau constitutionnel plusieurs catégories (mesure de sûreté, mesures de police) qui, bien que privatives de liberté, échappent aux principes constitutionnels de la loi pénale ${ }^{12}$.

Deuxièmement, tout en justifiant le transfert de compétence vers l'administration, le contrôle du Conseil constitutionnel ne pouvait pas être (ou en tout cas ne se voulait pas) aussi exigeant que dans le cadre de l'article 66 de la Constitution. Si le contrôle du Conseil constitutionnel avait été identique, cela aurait signifié que la nature des actes ou bien leurs conséquences sur la liberté fussent également identiques. Les différents degrés de contrôle étaient nécessaires pour maintenir, autant que justifier, la différence entre « privation » de liberté, d'une part, et « restriction » de liberté, d'autre part. Il va sans dire que le Conseil constitutionnel, pour maintenir cette distinction, s'est retrouvé parfois à quantifier, en heures, la limitation de liberté. Par exemple, la transformation de l'assignation à résidence administrative - mesure de police - en mesure privative de liberté en cas d'astreinte à domicile de plus de douze heures ${ }^{13}$.

Troisièmement, le Conseil constitutionnel s'est non seulement abstenu de contrôler les mesures préventives et privatives de liberté en elles-mêmes - d'en apprécier la " sévérité »- mais aussi d'intégrer dans le contrôle de ces mesures la question de leur environnement, c'est-à-dire, leur "nécessité». Si en matière pénale, bien qu'exceptionnellement ${ }^{14}$, le Conseil constitutionnel accepte de contrôler la sévérité ou la nécessité de la loi pénale - en s'appuyant sur les principes constitutionnels de la loi pénale (article 7, 8 et 9 de la Déclaration de 1789) - un tel contrôle est totalement exclu ailleurs. En dehors de la matière pénale, le contrôle du respect des droits et libertés se décline davantage en termes de conciliation et de proportionnalité (au sens large), soit un contrôle structurellement différent de celui de "sévérité » et de "nécessité " qu'exige la mesure privative de liberté. Ainsi, après avoir validé des mesures, que le Conseil constitutionnel qualifie lui-même d'attentatoires en elles-mêmes aux libertés ${ }^{15}$, sous l'angle de la conciliation, il était très difficile pour lui d'affirmer que les mesures de sûreté contestées en l'espèce, étaient, en elles-mêmes, disproportionnées. Cela serait revenu à entrer dans une logique permettant de juger, si l'on s'autorise un regard rétrospectif, que les assignations à résidence administratives, bien que validées, étaient également potentiellement disproportionnées. Censurer, en revanche, les mesures de sûreté en question, en les qualifiant de "peine pénale ", revenait à défaire trente années de jurisprudence et de législation, ficelées de manière intensive pendant et après l'état d'urgence ${ }^{16}$.

Afin d'invalider les mesures de sûreté, en l'espèce, le Conseil constitutionnel devait donc contrôler la «sévérité " et la «nécessité » de ces mesures «restrictives » de liberté, tout en refusant de les faire entrer dans le champ pénal, c'est-à-dire sans les qualifier de « privatives » de liberté et, par conséquent, sans leur appliquer les articles 66 de la Constitution ainsi que les articles 7, 8 et 9 de la Déclaration de 1789. Or, à l'impossible nul n'est tenu. La meilleure solution consistait alors à justifier le raisonnement par un "progrès » en matière de protection des droits et libertés. C'est 
ainsi que le Conseil constitutionnel transpose, pour la première fois, le considérant de principe de l'article 66 de la Constitution ${ }^{17}$, sans en mentionner - bien évidemment l'origine, au contrôle de la «liberté personnelle", telle que celle-ci découle (ou découlait) pourtant des articles 2 et 4 de la Déclaration de 1789 :

"Toutefois, bien que dépourvue de caractère punitif, elle doit respecter le principe, résultant des articles 2, 4 et 9 de la Déclaration de 1789, selon lequel la liberté personnelle ne saurait être entravée par une rigueur qui ne soit nécessaire. Il incombe au législateur d'assurer la conciliation entre, d'une part, la prévention des atteintes à l'ordre public et, d'autre part, l'exercice des droits et libertés constitutionnellement garantis. Au nombre de ceux-ci figurent la liberté d'aller et de venir, composante de la liberté personnelle, le droit au respect de la vie privée protégé par l'article 2 de la Déclaration de 1789 et le droit de mener une vie familiale normale qui résulte du dixième alinéa du Préambule de la Constitution du 27 octobre 1946. Les atteintes portées à l'exercice de ces droits et libertés doivent être adaptées, nécessaires et proportionnées à l'objectif de prévention poursuivi (nous soulignons) ».

Premièrement, cela signifie, plus précisément, que le Conseil constitutionnel accepte dorénavant de soumettre toutes les mesures « restrictives de liberté »- mesures de police préventives ou mesures de sûreté et d'autres à venir - au contrôle exercé dans le cadre de l'article 66 de la Constitution donc des mesures privatives de liberté.

Deuxièmement, le Conseil constitutionnel, afin de justifier ce transfert de contrôle, indique que la liberté personnelle découle désormais non seulement des articles 2 et 4 de la Déclaration mais aussi de son article 9 ; article dont la rédaction a inspiré celle du considérant de principe tel que formulé par le Conseil constitutionnel lorsque l'article 66 de la Constitution s'applique ${ }^{18}$. La référence à l'article 9 de la Déclaration est délicate car, si l'on suit la jurisprudence habituelle du Conseil constitutionnel, elle devrait emporter qualification en mesure pénale des dispositions contestées, qualification qu'il rejette sèchement par ailleurs.

Le Conseil constitutionnel réitère dans cette perspective sa décision «Rétention de sûreté » en évitant de répéter les mêmes « erreurs ». Pour rappel, dans cette décision, le Conseil constitutionnel avait, en toute souveraineté, refusé de qualifier la rétention de sûreté de peine pénale, tout en la qualifiant, avec la même souveraineté, de mesure privative de liberté. Il avait ainsi accepté de lui appliquer le principe de nonrétroactivité de la loi pénale ${ }^{19}$. Dans la décision du 7 août 2020 , il refuse de qualifier la mesure de sûreté de peine pénale (confirmation). Il ne la qualifie pas davantage de mesure privative de liberté (revirement). Il ne lui applique pas un principe constitutionnel de la loi pénale (revirement également). Mais en choisissant de justifier le nouveau contrôle exercé dans le cadre de la liberté personnelle par la mobilisation de l'article 9 de la Déclaration de 1789, sans reconnaitre la coloration pénale des mesures de sûreté, le Conseil constitutionnel finit par "dépénaliser » la protection, jusqu'alors pénale, accordée par le même article de la Déclaration de 1789.

21 Les principes constitutionnels de la loi pénale, après avoir constitutionnellement " pénalisé » des matières, seraient-ils, eux-mêmes, en voie de " dépénalisation »? Dans ce cas, quel obstacle conceptuel le Conseil constitutionnel pourrait-il sérieusement avancer si, un jour, la possibilité de prononcer ce type de mesures « restrictives » de liberté, en plus des « sanctions ayant le caractère d'une punition », est revendiquée - au nom de l'efficacité par exemple - en faveur du Conseil de la concurrence ou bien en faveur d'autres administrations et juridictions, en dehors, a fortiori, du Code pénal et de 
celui de la procédure pénale ? Le Conseil constitutionnel se contentera-t-il simplement de son contrôle " exigeant » en matière de « liberté personnelle » et/ou des " garanties procédurales " devant le Conseil de la concurrence, le Conseil supérieur de l'audiovisuel, etc. ?

\section{IV/- Inquiétude sur la solution}

La solution se veut rassurante pour l'avenir car elle semble renforcer le contrôle des mesures "restrictives" de liberté menées dans le cadre de toute politique de prévention, bien qu'en matière de lutte contre le terrorisme, les mesures les plus graves aient déjà été validées. Dans ce cadre en particulier, le Conseil constitutionnel semble pouvoir simplement censurer, comme en l'espèce, la multiplication, sous d'autres appellations juridiques, des mesures déjà existantes.

Mais si la censure est rassurante, la décision est inquiétante dans ce qu'elle sousentend. En refusant de reconnaître le caractère répressif de telles mesures et de censurer la compétence de la juridiction régionale de la rétention de sûreté sur le fondement des principes constitutionnels de la loi pénale, si généreusement distribués ailleurs, le Conseil constitutionnel consolide, tout en proposant un contrôle approfondi, la dépénalisation des mesures privatives de liberté - ces mesures nécessaires à un mode particulier d'exercice du pouvoir qui est la répression préventive.

Conseil constitutionnel, décision $n^{\circ} 2020-805$ DC du 7 août 2020, Loi instaurant des mesures de sûreté à l'encontre des auteurs d'infractions terroristes à l'issue de leur peine.

Les Lettres « Actualités Droits-Libertés » (ADL) du CREDOF (pour s'y abonner) sont accessibles sur le site de la Revue des Droits de l'Homme (RevDH) - Contact

\section{NOTES}

1. Décision n²017-750 DC du 23 mars 2017, Loi relative au devoir de vigilance des sociétés mères et des entreprises donneuses d'ordre, cons. 13.

2. Décision $n^{\circ}$ 2018-729 QPC du 7 septembre 2018, Société Tel and Com [Sanction de la nullité d'un licenciement économique]; décision $\mathrm{n}^{\circ}$ 2018-766 QPC du 22 février 2019, Mme Sylviane D. [Majoration du dépôt de garantie restant dû à défaut de restitution dans les délais prévus]; décision $n^{\circ}$ 2018-767 QPC du 22 février 2019, Société ODDO BHF [Exclusion de l'assiette des cotisations sociales des actions attribuées gratuitement]. 
3. Décision $n^{\circ}$ 2017-634 QPC du 2 juin 2017, M. Jacques R. et autres [Sanction par l'AMF de tout manquement aux obligations visant à protéger les investisseurs ou le bon fonctionnement du marché].

4. Décision $\mathrm{n}^{\circ}$ 2018-703 QPC du 4 mai 2018, Société People and Baby [Pénalité pour défaut d'accord collectif ou de plan d'action relatif à l'emploi des salariés âgés].

5. Décision n²016-554 QPC du 22 juillet 2016, M. Gilbert B. [Amende pour défaut de déclaration de comptes bancaires ouverts, utilisés ou clos à l'étranger II].

6. Décision $n^{\circ}$ 2013-341 QPC du 27 septembre 2013, M. Smaïn Q. et autres [Majoration de la redevance d'occupation du domaine public fluvial pour stationnement sans autorisation].

7. Décision n²017-634 QPC du 2 juin 2017, M. Jacques R. et autres [Sanction par l'AMF de tout manquement aux obligations visant à protéger les investisseurs ou le bon fonctionnement du marché].

8. Décision $n^{\circ}$ 2017-675 QPC du 24 novembre 2017, Société Queen Air [Procédure de sanction devant l'autorité de contrôle des nuisances aéroportuaires].

9. Décision n² 2015-489 QPC du 14 octobre 2015, Société Grands Moulins de Strasbourg SA et autres [Saisine d'office et sanctions pécuniaires prononcées par le Conseil de la concurrence].

10. Décision $n^{\circ} 2000-433$ DC du 27 juillet 2000, Loi modifiant la loi $n^{\circ}$ 86-1067 du 30 septembre 1986 relative à la liberté de communication.

11. Marion Lacaze, «Le droit des étrangers, "laboratoire » du droit de la prévention du terrorisme », RDLF 2019 chron. n41, http://www.revuedlf.com/droit-penal/le-droit-desetrangers-laboratoire-du-droit-de-la-prevention-du-terrorisme/.

12. Pour un exemple récent, décision $n^{\circ}$ 2018-770 DC du 6 septembre 2018, Loi pour une immigration maitrisée, un droit d'asile effectif et une intégration réussie, cons. 69 : « Ni les dispositions contestées $\mathrm{du} \mathrm{b} \mathrm{du} 8^{\circ}$ de l'article 29 , relatives à la durée maximale du placement en rétention administrative, ni celles du $2^{\circ}$ de l'article 23 , relatives aux cas de risques de fuite susceptibles de justifier le prononcé d'une obligation de quitter le territoire sans délai et, sous certaines conditions, le placement en rétention administrative, n'instituent une sanction ayant le caractère d'une punition. Par suite, le grief tiré de la méconnaissance de l'article 8 de la Déclaration de 1789 doit être écarté. » (nous soulignons).

13. Décision n² 2015-527 QPC du 22 décembre 2015, M. Cédric D. ; décision n²017-691 QPC du 16 février 2018, Farouk B.

14. Vincent Sizaire, « Pas plus qu'il n'est nécessaire ", La Revue des droits de l'homme [En ligne], Actualités Droits-Libertés, mis en ligne le 04 octobre 2020, URL : http://journals.openedition.org/ revdh/10471; DOI : https://doi.org/10.4000/revdh.10471.

15. Décision $n^{\circ}$ 2017-691 QPC du 16 Février 2018, Farouk B. Selon le Conseil constitutionnel les assignations à résidence administratives « en tant que telles, portent une atteinte à la liberté d'aller et de venir, au droit au respect de la vie privée et au droit de mener une vie familiale normale » (nous soulignons).

16. Vincent Sizaire, « Pas plus qu'il n'est nécessaire ", op.cit.

17. Ce considérant est le suivant : «Aux termes de l'article 66 de la Constitution : « Nul ne peut être arbitrairement détenu. - L'autorité judiciaire, gardienne de la liberté individuelle, assure le respect de ce principe dans les conditions prévues par la loi ». La liberté individuelle, dont la protection est confiée à l'autorité judiciaire, ne saurait être entravée par une rigueur non nécessaire. "

18. «Tout homme étant présumé innocent jusqu'à ce qu'il ait été déclaré coupable, s'il est jugé indispensable de l'arrêter, toute rigueur qui ne serait pas nécessaire pour s'assurer de sa personne doit être sévèrement réprimée par la loi »(nous soulignons).

19. Décision $\mathrm{n}^{\circ}$ 2008-562 DC du 21 février 2008, Loi relative à la rétention de sûreté et à la déclaration d'irresponsabilité pénale pour cause de trouble mental, cons. 9 et 10 . 


\section{ABSTRACTS}

Dans sa décision du 7 août 2020, le Conseil constitutionnel censure la mesure de sûreté prononcée à l'encontre d'un auteur d'infraction terroriste à l'issue de sa peine. Pour la première fois, il transpose le contrôle exercé dans le cadre des atteintes portées à la « liberté individuelle » à celui des atteintes portées à la « liberté personnelle ». En d'autres termes, il semblerait que, désormais, mesures « restrictives » et « privatives » de liberté soient soumises au même contrôle «serré » du Conseil constitutionnel. En arrière-plan cependant, la décision montre davantage ce que le Conseil constitutionnel consolide que ce qu'il censure.

\section{AUTHOR}

\section{PATRICIA RRAPI}

Maîtresse de conférences à l'Université Paris Nanterre 